\title{
Modalidades de despliegue de infraestructura en telecomunicaciones
}

\author{
Alexander Correa*
}

\begin{abstract}
RESUMEN
Este estudio está dirigido a investigar las decisiones de la firma sobre el grado de mejoras en calidad en la prestación de servicios de nueva generación cuando otras aproximaciones de despliegue de infraestructura, especialmente la coinversión, actúan como modos alternativos para la introducción de estos nuevos servicios al mercado. El modelo desarrollado en este estudio tiene en consideración la asimetría de las firmas. Este estudio muestra que la coinversión resulta en un mayor bienestar del consumidor que la regulación de costos totalmente distribuidos a pesar de que los niveles de inversión en mejoras que aumentan la calidad son más bajos. Sin embargo, comparado con el escenario de inversión individual, la coinversión produce un deterioro sobre las mejoras de calidad cuando la compartición de infraestructura induce una considerable cantidad de costos incrementales. El regulador debería monitorear los acuerdos de coinversión para evitar situaciones de colusión tácita especialmente cuando las firmas exhiben asimetría en costos en el despliegue de redes de nueva generación.
\end{abstract}

Palabras clave: despliegue de infraestructura, telecomunicaciones, coinversión, regulación.

Códigos JEL: L12, L51, L96

\section{Modalities of Deployment of Telecommunications Infrastructure}

\begin{abstract}
This study is aimed at investigating the firm's decisions about the degree of quality improvements for advanced technology services when other approaches to infrastructure deployment, especially co-investment, act as alternative ways to launch new services. The model developed in this study considers the asymmetry of firms. This study shows that co-investment results in greater consumer welfare than fully distributed cost regulation, even though investment levels in quality improvements are lower. However, compared to individual investment, co-investment produces a deterioration in quality improvements when infrastructure sharing induces a considerable amount of incremental costs. The regulator should monitor co-investment agreements to avoid situations of tacit collusion especially when firms exhibit cost asymmetry in the deployment of advanced technology.
\end{abstract}

Keywords: Infrastructure sharing, telecommunications, co-investment, regulation.

JEL Codes: L12, L51, L96

* Doctorando en Modelado en Gestión y Política Pública, Universidad Jorge Tadeo Lozano, Colombia. correa.alexander@gmail.com 


\section{INTRODUCCIÓN}

En el mercado de internet de banda ancha, la tendencia creciente es hacia el despliegue de redes de acceso de nueva generación, e.g. redes de fibra óptica, con altas capacidades para satisfacer la creciente demanda por internet de alta velocidad ${ }^{1}$. Adicional a ofrecer altas calidades en el servicio, los operadores de redes de telecomunicaciones pueden lograr ventajas en el mercado a través de reducciones en sus costos y diferenciando sus productos a partir de la adopción de nuevas tecnologías. Sin embargo, realizar inversiones en dichas tecnologías, para proporcionar servicios de alta calidad o de valor agregado, implica altas sumas de recursos financieros. Un operador de telecomunicaciones debe incurrir en altos costos de inversión para desplegar su propia infraestructura si decide entrar al mercado mediante un modelo en el cual construye su propia infraestructura. De acuerdo con las políticas de entrada facilitadas por los reguladores, un entrante puede elegir también competir con base en servicios y realizar un pago (precio de acceso) a la empresa establecida en retorno por arrendar su infraestructura. De acuerdo con la teoría de la escalera de inversión (Cave, 2006; Cave y Voselsang, 2003) este modo de entrada puede estimular la competencia en el mercado minorista cuando la entrada basada en infraestructura no es una opción factible para un entrante debido a la falta de recursos financieros y a la duplicación improductiva de infraestructura. Por otro lado, la conversión es una alternativa a la inversión convencional para acelerar el despliegue de redes de nueva generación, especialmente en áreas de baja demanda y altos costos de inversión. Los coinversionistas pueden de manera individual construir su propia infraestructura en áreas de alta demanda y ponerse de acuerdo en coinvertir en áreas de baja demanda para dar cumplimiento a las obligaciones de cobertura impuestas por el regulador. Adicionalmente, algunos coinversionistas tienen contratos para desplegar únicamente una pequeña proporción de infraestructura seleccionada, mientras que otros coinversionistas pueden ponerse de acuerdo en desplegar totalmente su infraestructura. Se afirma que el ahorro en costos es proporcional a la intensidad del despliegue de infraestructura (Beckman y Smith, 2005; Song, Zo y Lee, 2012).

La aproximación óptima para incentivar la inversión dirigida a mejorar la calidad de los servicios de telecomunicaciones, especialmente a través del despliegue de redes de nueva generación, ha sido un tema en debate por cerca de una década bajo el dilema existente entre eficiencia estática y eficiencia dinámica, tal como se ha discutido tanto en la literatura empírica como en la teórica. El concepto de desagregación del bucle local ha sido implementado de manera extensa con el fin de estimular la competencia basada en servicios, aunque se afirma que esta aproximación no promueve la inversión ni la

1 En el mercado de las telecomunicaciones móviles, la tecnología inalámbrica 2G ha sido gradualmente reemplazada por $3 \mathrm{G}$ y de manera más reciente con $4 \mathrm{G}$ con el prospecto de tecnologías superiores que permiten a los consumidores disfrutar no solo de servicios de voz, sino también de servicios de valor agregado tales como transferencia de datos y de internet en consonancia con la convergencia tecnológica. 
innovación en el largo plazo (Bacache, Bourreau y Gaudin, 2014; Briglauer, Ecker y Gugler, 2013; Crandall, Eisenach y Ingraham, 2013). Otras aproximaciones de despliegue de infraestructura han sido discutidas de manera crítica en el contexto de eficiencia dinámica. Los estudios relacionados examinan los efectos de la coinversión en diferentes aspectos. Muchos de ellos se enfocan solo en cómo la coinversión estimula la inversión bajo un escenario de simetría de las firmas (Cambini y Silvestri, 2013; Nitsche y Wiethaus, 2011). Sin embargo, las firmas de telecomunicaciones son usualmente asimétricas, especialmente en mercados incipientes con tasas de alto cambio tecnológico. Esto lleva a diferencias en la estructura de costos y en la demanda, discrepancias en la calidad del servicio, comportamientos predatorios por parte de las firmas dominantes y colusión que pueden tener efectos significativos sobre el bienestar social y el despliegue de tecnología avanzada hacia el futuro.

La evidencia empírica reciente también sustenta el hecho que la regulación del precio de acceso y la desagregación obligatoria del bucle local no estimula el despliegue de redes de nueva generación (Bacache et al., 2014; Bouckaert, van Dijk y Verboven, 2010; Briglauer et al., 2013; Briglauer, Gugler y Haxhimusa, 2015; Crandall et al., 2013; Grajek y Röller, 2012). Briglauer et al. (2013), usando datos de los miembros de la Unión Europea para los ańos 2005 y 2011, revelaron que la competencia bajo desegregación obligatoria del bucle local tiene un impacto adverso sobre el despliegue de fibra óptica como servicio de internet de banda ancha. Este hallazgo también es confirmado con evidencia de países de la OCDE. Bouckaert et al. (2010), usando datos de veinte países de la OCDE en el periodo de 2003 a 2008, encuentran que la penetración de banda ancha es promovida mediante la competencia intraplataformas. Por lo tanto, se sugiere que la competencia intra plataforma es el modo eficiente de expandir la penetración de banda ancha. Briglauer et al. (2015) utilizan datos al nivel de la firma y entrantes de países europeos en el periodo de 2003 a 2012. Ellos proporcionan evidencia significativa que la competencia basada en infraestructura tiene un efecto positivo sobre la inversión tanto de empresas existentes como de entrantes.

Los reguladores enfrentan el desafío de nuevos modelos regulatorios para promover el despliegue redes de nueva generación en el mercado de internet de banda ancha cuando sus consideraciones van más allá de un marco estático. Aunque precios basados en costos pueden disminuir la inversión en redes de nueva generación desde una perspectiva dinámica, otras alternativas regulatorias requieren estar fundamentadas en estudios que justifiquen su utilización. La desregulación o la autorregulación pueden ser aproximaciones correctas bajo ciertas circunstancias; sin embargo, es necesario para los reguladores monitorear cuidadosamente el modo en que se desarrolla la competencia en redes de nueva generación. En los Estados Unidos, la desregulación ha sido implementada en el mercado de banda ancha con el propósito de facilitar el despliegue de redes de nueva generación (Cambini y Jiang, 2009). 
En ese orden de ideas, este estudio está dirigido a investigar las decisiones de la firma para invertir en mejoras que aumentan la calidad de las redes de nueva generación cuando otras aproximaciones de despliegue de infraestructura, especialmente la coinversión, actúan como modos alternativos para el lanzamiento de nuevos servicios. Además de diferentes acuerdos de despliegue de infraestructura, el modelo desarrollado en este estudio tiene en consideración la asimetría de las firmas, donde la estructura de costos de las mismas es diferente entre ellas. Este estudio muestra que la coinversión resulta en un mayor bienestar del consumidor que la regulación de costos totalmente distribuidos a pesar de que los niveles de inversión en mejoras de calidad son más bajos. Sin embargo, comparado con la figura de inversión individual, la coinversión produce deterioren las mejoras en calidad cuando el despliegue de infraestructura implica un aumento considerable de costos. Por lo tanto, en contraste a lo que las firmas de telecomunicaciones usualmente manifiestan ${ }^{2}$, puede que la coinversión no sea el modo más apropiado para estimular el despliegue de tecnología avanzada en algunas situaciones. El regulador debería monitorear detalladamente los acuerdos de coinversión para evitar situaciones de colusión tácita especialmente cuando las firmas exhiben asimetría en costos en el despliegue de redes de nueva generación.

Este estudio está organizado como sigue: En la sección 2 se hace una revisión breve de la literatura. El modelo se introduce en la sección 3. La sección 4 presenta el análisis de los resultados de equilibrio y la comparación entre la coinversión y otras aproximaciones de despliegue de infraestructura respectivamente. Las implicaciones de política se plantean en la sección 5. Finalmente, las conclusiones se presentan en la sección 6 .

\section{REVISIÓN LITERATURA}

La literatura acá revisada enfatiza que el precio de acceso tiene un efecto crucial sobre el nivel de la competencia en el mercado cuando se opera en un esquema de despliegue de infraestructura bajo la modalidad de entrada basada en servicios. El establecido, quien ha construido su propia infraestructura, puede aumentar sus beneficios en el mercado mayorista fijando un precio de acceso alto lo que pondría al entrante, quien opera arrendando la infraestructura del establecido para ofrecer sus propios servicios, en desventaja. Un aumento en el precio mayorista provoca que el precio minorista aumente y finalmente se reduzca la competencia (Bourreau, Cambini y Dogan, 2012; Bourreau, Cambini y Hoernig, 2015). Mientras tanto, precios de acceso bajos pueden estimular la competencia facilitando que más entrantes ingresen de manera factible al mercado. Así, en términos de eficiencia estática, la regulación de los precios de acceso basados en costos tiende a ser necesaria.

\footnotetext{
2 Ver http://www.telegraph.co.uk/business/2017/08/22/vodafone-talks-openreach-big-investment-ukbroadband/
} 
Sin embargo, desde una perspectiva dinámica, el precio de acceso tiene un efecto significativo sobre la inversión en redes de nueva generación. Precios de acceso bajos pueden desestimular las empresas con infraestructura propia a invertir en mejores tecnologías ya que dichos precios bajos no les permite extraer beneficios suficientes en el marco de acuerdos regulados de despliegue de infraestructura (Bourreau et al., 2015; Cambini y Silvestri, 2013; Cambini y Valletti, 2003; Kotakorpi, 2006; Nitsche y Wiethaus, 2011; Vareda, 2010). De otro lado, precios de acceso altos pueden incentivar la inversión en el despliegue de nuevas tecnologías (Vareda, 2010), y también pueden hacer que los entrantes construyan su propia infraestructura en vez de elegir entrar al mercado arrendando infraestructura. Nitsche y Wiethaus (2011) compararon el resultado para cuatro aproximaciones diferentes de despliegue de infraestructura, incluyendo la opción de no hacer nada desde el punto de vista regulatorio, el régimen de costos totalmente distribuidos, compartición de riesgo mediante coinversión y regulación de costos incrementales de largo plazo (LRIC). Estos autores demuestran que la entrada basada en servicios bajo una regulación de precios de acceso orientados a costos (LRIC) lleva a un despliegue bajo de redes de nueva generación al igual que reduce el bienestar del consumidor. Adicionalmente, la compartición de riesgo mediante coinversión lleva a un bienestar del consumidor alto.

Comparado con el escenario de inversión individual, se afirma que la coinversión estimula la inversión en servicios altos en calidad (Cambini y Silvestri, 2013; Nitsche y Wiethaus, 2011) y aumenta el bienestar social (Cambini y Silvestri, 2013; Foros, Hansen y Sand, 2002; Nitsche y Wiethaus, 2011). Adicionalmente, la coinversión proporciona un incentivo a los coinversionistas a expandir su cobertura bajo algunas circunstancias en ciertas regiones o zonas geográficas (Bourreau et al., 2015; Krämer y Vogelsang, 2014). Cambini y Silvestri (2013) examinan el mercado de banda ancha en un marco de competencia a la Cournot en una sola región bajo tres diferentes alternativas de inversión: (1) compartición de infraestructura con un precio de acceso orientado a costos cobrado a la empresa que arrienda infraestructura para entrar al mercado, (2) despliegue de infraestructura básica sin pago adicional y (3) un acuerdo de joint venture con pago adicional. Estos autores encuentran que el acuerdo de joint venture genera el mayor incentivo para invertir en nuevas tecnologías. No obstante, a pesar de lograr los niveles más altos de despliegue de tecnología, el acuerdo de joint venture puede reducir la competencia porque las empresas del joint venture pueden coludir para fijar pagos recíprocos por encima del costo. Como resultado, el acuerdo de joint venture con la colusión de pagos y el régimen de precios de acceso orientados a costos junto con el menor incentivo para invertir resultan ambos en menor excedente del consumidor y menor bienestar social que lo que se da bajo la figura básica de inversión.

Adicionalmente, Bourreau, Cambini y Hoerning (2012) analizan el efecto de la coinversión sobre las decisiones de la firma de expandir su cobertura. En ese contexto, se asume que la estructura de costos de la inversión puede cambiar después del acuerdo 
de coinversión en línea con los beneficios o costos adicionales producto de la figura que se use para desplegar infraestructura. Los costos expost del despliegue de infraestructura pueden caer debido a una reducción en el riesgo financiero. El costo de inversión probablemente se incremente debido al costo adicional de utilizar equipo técnico para operar dos redes sobre la infraestructura compartida. De manera similar al presente estudio, estos autores concluyen que la coinversión incentiva la expansión de la cobertura cuando la diferenciación de servicios y los beneficios producto de la reducción de costos son lo suficientemente significativos. Adicionalmente, también se encuentra que precios de acceso altos incentivan a que un entrante negocie un acuerdo de coinversión en vez de elegir entrar al mercado arrendando infraestructura. El efecto positivo de la coinversión sobre la expansión de cobertura también es confirmado por la más reciente evidencia experimental (Krämer y Vogelsang, 2016).

La literatura existente muestra apoyo por la figura de coinversión como herramienta para promover el despliegue de redes de nueva generación. Sin embargo, el presente estudio advierte que, en algunas situaciones, puede que la coinversión no sea la figura apropiada que permita acelerar la inversión en mejoras que aumenten la calidad de las redes de nueva generación.

\section{MODELO}

Este estudio se concentra en servicios de telecomunicaciones, especialmente servicios de internet de banda ancha con inversiones significativas en redes de nueva generación, las cuales pueden representar una carga onerosa tanto para los proveedores de red establecidos como para los que recién entran al mercado. Existen dos firmas invirtiendo en tecnologías de nueva generación en una región determinada. La nueva tecnología cambia de acuerdo con el nivel de calidad que las dos firmas eligen ${ }^{3}$.

\section{Costo}

Se asume que el costo de inversión del despliegue de las redes de nueva generación ${ }^{4}$ cuando la firma $i$ elige desplegar sus servicios avanzados a un nivel dado de calidad $s_{i}$ es

$$
c_{i}\left(s_{i}\right)=\frac{\alpha_{i}}{2} s_{i}^{2}
$$

\footnotetext{
3 Este estudio está basado en competencia en el mercado de banda ancha de internet. Las firmas han estado compitiendo por mayor calidad a través de redes de nueva generación tales como fibra al hogar. Sin embargo, este modelo puede ser aplicado a la siguiente generación de redes móviles las cuales se dirigen a tecnologías $5 \mathrm{G}$ o más avanzadas con la convergencia tecnológica. Otra aplicación son las mejoras en calidad sobre la tecnología existente. La velocidad de transferencia de datos, una reducción en la congestión de tráfico de datos, conectividad sostenible y servicio al consumidor pueden ser tomadas como consideraciones para el propósito de planeación estratégica.

4 El costo de inversión es el costo fijo involucrado con el despliegue de servicios avanzados tales como el costo fijo de instalaciones y operación de acuerdo con el nivel seleccionado de mejora de calidad.
} 
$c_{i}^{\prime}\left(s_{i}\right)>0$ y $c_{i}^{\prime \prime}\left(s_{i}\right)>0$. $\alpha_{i}$ es un parámetro de costos; $\alpha_{i}>0$ y $s_{i} \geq 0$, donde $i \in\{1,2\}^{5}$. Por lo tanto, el costo de inversión es estrictamente creciente en $s$. Por simplicidad, los costos marginales de producción son normalizados a cero para ambas firmas ${ }^{6}$. En su lugar, este estudio enfatiza la asimetría en la estructura de costos de inversión donde la firma 1 tiene un costo menor que la firma $2^{7}$. $\alpha_{1}<\alpha_{2}$ y $\Delta_{\alpha}$ representa un parámetro de asimetría de costos; $\Delta_{\alpha}=\frac{\alpha_{2}}{\alpha_{1}}>1$.

\section{Demanda}

Siguiendo a Foros (2004) y Kotakorpi (2006), los consumidores representativos están uniformemente distribuidos con diferentes disposiciones a pagar por el servicio básico en un continuo de $(-\infty, a]$. Estos obtienen una utilidad adicional proveniente de las mejoras de calidad. Algunos consumidores que tienen valoraciones bajas del servicio no se suscriben al mismo. Bajo el supuesto de demanda unitaria ${ }^{8}$, la valoración de un consumidor representativo por el servicio de la firma $i$ esta dado por

$$
v+S\left(s_{i}\right)
$$

$v$ es la disposición a pagar por el servicio básico, que varía en el intervalo $(-\infty, a]$ entre consumidores. $S\left(s_{i}\right)$ es la utilidad adicional producto de las mejoras de calidad del servicio de la firma $i$ donde la firma $i$ elige el grado de la mejora de calidad al nivel de $s_{i}$; $s_{i} \geq 0 ; i \in\{1,2\}$.

\footnotetext{
5 Para actualizar el servicio básico a un servicio avanzado, las firmas necesitan invertir adicionalmente en infraestructura para facilitar las nuevas tecnologías. En adición, el costo de inversión del despliegue de tecnología avanzada puede variar entre áreas con diferente perfil geográfico, naturaleza de la demanda y densidad poblacional. Sin embargo, este modelo asume una única región y omite los temas de cobertura.

6 En los mercados de telecomunicaciones, la infraestructura para mejorar la calidad involucra una enorme cantidad de costos fijos en comparación con la cantidad casi despreciable de costos marginales correspondientes a la unidad de demanda o suscriptor. Así, por simplicidad, se asume que el costo marginal es constante sin importar el nivel de mejoras en calidad. No obstante, las decisiones de las firmas sobre el grado de las mejoras de calidad pueden tener un impacto sobre la estructura del costo de producción variable y por lo tanto del costo de producción marginal. Por ejemplo, la tecnología avanzada puede ser desarrollada para atender una mayor calidad de servicios de telecomunicaciones, y también puede reducir los costos de producción asociados.

Este modelo está desarrollado sobre el supuesto de información perfecta sobre la estructura de costos de las firmas. Ambas firmas tienen información completa sobre la estructura de costos de cada una. El costo del establecido puede ser observado a través de su comportamiento estratégico y su obligación de revelar su estructura de costos al regulador. De igual forma, la reputación del entrante como una corporación internacional o como un proveedor existente en otras regiones puede ser una seńal de la estructura de costos del entrante en esta región.

8 El supuesto de demanda unitaria es razonable en el sector de telecomunicaciones. Por ejemplo, en el mercado de internet de banda ancha, una familia elige establecer una conexión de internet con un proveedor de internet que ofrece un uso ilimitado de internet a cambio de un cargo fijo mensual. También se observa en el mercado móvil donde los operadores de red móviles ofrecen un paquete mensual de servicios móviles que incluyen minutos, mensajes de texto e internet.
} 
Si la firma $i$ decide no mejorar la calidad de su servicio básico, $s_{i}=0$ y $S\left(s_{i}\right)=0$. Para simplificar el modelo, todos los consumidores tienen utilidad adicional homogénea producto de las mejoras de la calidad del servicio en la siguiente forma ${ }^{9}$

$$
S\left(s_{i}\right)=\omega s_{i}
$$

con el parámetro $\omega>0 \theta \omega^{10}$.

Este modelo está basado en un modelo de competencia a la Cournot. La firma $i$ ofrece la mejora en calidad $\left(s_{i}\right) ; i \in\{1,2\}$. Así, la firma probablemente elige diferentes niveles de mejoras en calidad de lo que lo hace su competencia. Un consumidor representativo evalúa los dos servicios. Este se suscribirá a la firma que ofrece mayor utilidad neta cuando su utilidad es todavía positiva. Por ejemplo, el consumidor comprará el servicio a la firma $i$ en vez que a la firma $j$ cuando se cumple la siguiente condición.

$$
v+\omega s_{i}-p_{i}>v+\omega s_{j}-p_{j}
$$

donde $p_{i}$ (respectivamente $p_{j}$ ) es el precio minorista del servicio avanzado de la firma $i$ (respectivamente $j$ ) con el grado de mejora de calidad de $s_{i}$ (respectivamente $s_{j}$ ); $i, j \in\{1,2\}$ e $i \uparrow j$.

El precio ajustado por calidad de la firma $i$ es

$$
P_{i}=p_{i}-\omega s_{i} ; i \in\{1,2\}
$$

Bajo este marco de competencia, la firma $j$ será obligada a salir del mercado si su precio ajustado por calidad $\left(P_{j}\right)$ es más alto que el de la firma $i$ para todo consumidor. Esto es porque cada consumidor dará una mayor valoración al servicio de la firma $i$ que al servicio de la firma $j$. Este estudio se dirige a analizar el equilibrio compartido de mercado donde ambas firmas están activas en el mercado. Por lo tanto, los precios ajustados por calidad de los servicios activos de ambas firmas deben ser iguales, de otro modo la

9 Este modelo asume que la forma de la utilidad aditiva del servicio de mejoras en calidad afecta a todos los consumidores representativos en exactamente el mismo modo. Adicionalmente, la teoría de elección discreta de diferenciación del producto incorpora las características del producto tales como calidad dentro de la función de utilidad en forma aditiva.

10 Tal y como asumen Mussa y Rosen (1978) y Valletti (2000) a la luz de diferenciación vertical, la función de utilidad de un consumidor por un producto particular se deriva simplemente de multiplicar el parámetro de prueba y el nivel de calidad. En otras palabras, un consumidor representativo tiene una utilidad marginal de calidad igual a $\theta$.

Adicionalmente, en la industria de telecomunicaciones, la mayoría de los consumidores están preocupados por la calidad. En el mundo real, las firmas que invierten en tecnologías de alta calidad pueden también operar un servicio estándar o de baja calidad en la misma plataforma para poder competir en un mercado nicho, perfilando usuarios que eligen un servicio de calidad inferior. Por lo tanto, este estudio reduce los diferentes tipos de consumidores a un solo grupo de consumidores con igual parámetro de prueba $\omega$, y la utilidad adicional sigue una forma funcional lineal que corresponde a la utilidad marginal constante de la calidad. Este estudio excluye el tema clásico de la diferenciación vertical. 
firma con un precio mayor seria excluida del mercado. El precio ajustado por calidad que satisface el equilibrio compartido de mercado $(P)$ se muestra a continuación

$$
P=P_{i}=P_{j}=p_{i}-\omega s_{i}=p_{j}-\omega s_{j}
$$

En este contexto, el nivel de producción de la industria $(Q)$ equivale a la capacidad instalada de la misma que al igualarse con la demanda resulta en el número de consumidores que se suscriben a cualquier servicio. Un consumidor cuya utilidad es más alta que o igual al precio ajustado por calidad $(P)$ participará en el mercado $(v-P \geq 0)$. De acuerdo con el supuesto de población uniformemente distribuida y demanda unitaria, el número total de suscriptores es $a-P$ al precio ajustado por calidad $P$. Es implícito que la función de demanda agregada es $Q=a-P$ y $Q=q_{1}+q_{2}$, donde $q_{1}$ y $q_{2}$ son los niveles de producción que las firmas 1 y 2 , respectivamente ${ }^{11}$. Así, la función de demanda inversa enfrentada por la firma $i$ se deriva a continuación.

$$
p_{i}=a+\omega s_{i}-q_{1}-q_{2} \text { donde } i \in\{1,2\}
$$

el precio de la firma $i\left(p_{i}\right)$ corresponde directamente a su elección de mejoras en calidad $\left(s_{i}\right)$ y nivel de producción $\left(q_{i}\right)^{12}$.

Este estudio examina los efectos de diferentes modalidades de despliegue de infraestructura sobre las decisiones de la firma para mejorar los niveles de calidad en redes de nueva generación. El modelo está diseńado para capturar la competencia con respecto a diferentes aproximaciones de despliegue de infraestructura. En este estudio, hay tres aproximaciones prácticas, introducidas en los siguientes casos.

11 Para invertir en nueva infraestructura de telecomunicaciones, la capacidad debe ser planeada junto con la estrategia de negocios. Por ejemplo, las firmas deciden sobre la ubicación del acceso de red y la capacidad para servir a las familias objetivo con sus servicios de internet de banda ancha. Otro ejemplo es el de los operadores móviles que planifican la asignación de números móviles realizada por el regulador.

${ }^{12}$ La función de demanda agregada en una región determinada está dada por $Q=a-P$. Despues de reemplazar $Q \operatorname{con} q_{1}+q_{2}$ y $P$ con $p_{i}-\omega s_{i}$

$$
\text { Así, } p_{i}=a+\omega s_{i}-q_{1}-q_{2}
$$

$$
q_{1}+q_{2}=a-p_{i} s_{i}
$$

En este contexto de competencia a la Cournot, las firmas deciden sobre las mejoras en calidad en la etapa de inversión, para después elegir sus niveles de producción (el número de suscriptores a ser atendidos). Suponiendo que la etapa de inversión es exógena, a un par dado de niveles de mejoras en calidad de las firmas, estas eligen sus propios niveles de producción lo cual lleva a precio ajustado por calidad que equilibra el mercado (P) y por lo tanto sus precios minoristas (cargos por suscripción) deben corresponder al precio ajustado por calidad en el equilibrio de mercado compartido. Cuando la etapa de inversión es endógena, las firmas estratégicamente planean sus mejoras en calidad. Sus precios minoristas deben corresponder a las decisiones de las firmas sobre el grado de mejoras en calidad y los niveles de producción en el equilibrio de mercado compartido. 


\section{Caso 1: Las dos firmas con infraestructura propia invierten de manera separada en mejoras en calidad (inversión individual).}

Se asume que las dos firmas son asimétricas en su estructura de costos. Ellas deciden invertir separadamente sin desplegar infraestructura conjuntamente y maximizan sus propios beneficios. Las funciones de beneficios son

$$
\begin{gathered}
\pi_{N 1}=\left(a+\omega s_{N 1}-q_{N 1}-q_{N 2}\right) q_{N 1}-\frac{\alpha_{1}}{2} s_{N 1}^{2} \\
\pi_{N 2}=\left(a+\omega s_{N 2}-q_{N 1}-q_{N 2}\right) q_{N 2}-\frac{\alpha_{2}}{2} s_{N 2}^{2}
\end{gathered}
$$

donde $Q_{N}=q_{N 1}+q_{N 2}$, donde $N$ se refiere al escenario de inversión individual.

\section{Tiempo del juego}

Este modelo está diseñado en un marco estático. Cada firma toma una decisión sobre el grado en que se mejora la calidad del servicio (redes de nueva generación) en el siguiente juego de dos etapas.

Etapa 1 Las firmas de manera simultánea eligen el grado de mejora de calidad $\left(s_{N 1}, s_{N 2}\right)$.

Etapa 2 Las firmas de manera simultánea eligen sus niveles de servicio $\left(q_{N 1}, q_{N 2}\right)$.

\section{Caso 2: Las dos firmas coinvierten}

Después del acuerdo de coinversión, ambas firmas eligen de manera conjunta el grado de mejora de calidad del servicio $\left(s_{C}\right)^{13}$. La firma de costo bajo despliega su infraestructura en conjuntamente con su rival. La estructura de costos puede cambiar después de acomodarse a las operaciones de las dos firmas en vez de una sola firma. $C_{\text {compartición }}$ es el costo de inversión después del acuerdo de despliegue mutuo de infraestructura, el cual facilita los servicios de ambas firmas usando la infraestructura de la firma de costo bajo.

$$
C_{\text {compartición }}\left(s_{c}\right)=\frac{\varnothing \alpha_{1}}{2} s_{C}^{2}
$$

$\varnothing$ es un parámetro positivo de ajuste de costos de despliegue conjunto de infraestructura. $\varnothing=1$, cuando la infraestructura puede ser desplegada conjuntamente sin ningún costo adicional. $\varnothing>1$ cuando las firmas incurren en costos adicionales de equipamiento y operación para poder facilitar que los dos servicios funcionen sobre la infraestructura desplegada conjuntamente. $\varnothing<1$ cuando los ahorros en costos del despliegue conjunto

\footnotetext{
13 Se asume que las preferencias del consumidor por servicios mejorados por calidad ofrecidos por ambas firmas no son diferentes porque ambos servicios operan sobre la misma infraestructura compartida sobre el supuesto de no preferencia por una firma especifica.
} 
de infraestructura sobrepasan los costos adicionales ${ }^{14}$. Las dos firmas negocian la coinversión para llegar a un acuerdo sobre la base de una solución de Nash. Después de llegar a un acuerdo exitoso de coinversión, las firmas eligen mutuamente el grado en que se mejorará la calidad del servicio $\left(s_{C}\right)$ y acuerdan dividir el costo total de inversión. $\beta$ es la parte del costo total de inversión que la firma 1 paga y la firma 2 asume el resto, $1-\beta$.

Las funciones de beneficios son

$$
\begin{gathered}
\pi_{C 1}=\left(a+\omega s_{C}-q_{C 1}-q_{C 2}\right) q_{C 1}-\beta \frac{\varnothing \alpha_{1}}{2} s_{C}^{2} \\
\pi_{C 1}=\left(a+\omega s_{C}-q_{C 1}-q_{C 2}\right) q_{C 2}-(1-\beta) \frac{\varnothing \alpha_{1}}{2} s_{C}^{2}
\end{gathered}
$$

donde $Q_{C}=q_{C 1}+q_{C 2}$, donde $C$ se refiere al escenario de coinversión

\section{Tiempo del juego}

Ambas firmas llegan a un acuerdo de despliegue conjunto de infraestructura sobre el nivel en que se mejorara conjuntamente la calidad del servicio $\left(s_{C}\right)$ y la división del costo total de inversión $(\beta)$ siguiendo una solución de Nash en la etapa 1. A continuación, las firmas entran en la etapa 2 en un marco competencia a la Cournot en el mercado minorista.

Etapa 1 Las firmas negocian sobre el nivel en el que se mejorara la calidad $\left(s_{C}\right)$ y $(\beta)$ sobre la base de una solución de Nash.

Etapa 2 Las firmas eligen de manera simultánea sus propios niveles de servicio

\section{Caso 3: Acceso a infraestructura bajo un régimen de costos totalmente distribuidos}

El entrante con costos más altos decide usar la infraestructura del establecido para proveer su servicio en la región en vez de construir su propia infraestructura. El establecido está regulado para que proporcione acceso de red al entrante de acuerdo con un precio de acceso orientado a costos. El pago se calcula sobre la base de la metodología de costos totalmente distribuidos, y consecuentemente los costos de inversión para mejorar

\footnotetext{
${ }^{14}$ Para garantizar la existencia de una negociación exitosa sobre la coinversión, $\varnothing>0$ y $\varnothing$ no deberia ser muy grande para poder garantizar que ambas firmas asimétricas se pongan de acuerdo en compartir el costo de inversión; i.e. la proporción de equilibrio del costo total de inversión de la firma de costo bajo es positivo, $\beta^{*}>0$. Cuando la compartición de infraestructura genera un mayor costo de inversion ( $\varnothing$ es muy grande), es más probable que $\beta^{*} \leq 0$ porque $\frac{\partial \beta^{*}}{\partial \varnothing}<0$. Si $\beta^{*} \leq 0$, la firma de costo bajo no se embarca en la construcción de su propia infraestructura y el acuerdo de compartición de infraestructura usando la tecnología de la firma de costo bajo no ocurre. Consecuentemente, el equilibrio bajo coinversión no existe en este caso.
} 
la calidad del servicio se comparten dependiendo del uso de la infraestructura ${ }^{15}$. En este caso, el establecido con menores costos (firma 1) decide de manera unilateral sobre el grado de mejoras en calidad $s_{F}$ para poder maximizar su propio beneficio en respuesta a este régimen regulatorio ${ }^{16}$.

Las funciones de beneficios son

$$
\begin{array}{r}
\pi_{F 1}=\left(a+\omega s_{F}-q_{F 1}-q_{F 2}\right) q_{F 1}-\frac{\varnothing \alpha_{1}}{2} s_{F}^{2}+\left(\frac{\varnothing \alpha_{1}}{2} s_{F}^{2}\right)\left(\frac{q_{F 2}}{Q_{F}}\right) \\
\pi_{F 2}=\left(a+\omega s_{F}-q_{F 1}-q_{F 2}\right) q_{F 2}-\left(\frac{\varnothing \alpha_{1}}{2} s_{F}^{2}\right)\left(\frac{q_{F 2}}{Q_{F}}\right)
\end{array}
$$

donde $Q_{F}=q_{F 1}+q_{F 2}$, donde $F$ se refiere a costos totalmente distribuidos

El entrante basado en servicios paga el total del cargo de acceso de $\left(\frac{\varnothing \alpha_{1}}{2} s_{F}^{2}\right)\left(\frac{q_{F 2}}{Q_{F}}\right)$ al establecido basado en infraestructura de acuerdo con la proporción de uso.

\section{Tiempo del juego}

$\mathrm{Al}$ ser el establecido de costo bajo, la firma 1 proporciona acceso de red al entrante basado en servicios en el siguiente juego de dos etapas.

Etapa 1 La firma 1 decide sobre el grado en que se mejorará la calidad $\left(s_{F}\right)$ para maximizar sus propios beneficios.

Etapa 2 Las dos firmas de manera simultánea eligen sus niveles de provisión del servicio

\section{Excedente del consumidor}

El excedente del consumidor puede ser calculado de la siguiente manera

$$
C S_{k}=\left(\frac{1}{2}\right)\left(q_{k 1}+q_{k 2}\right)^{2}
$$

$k$ denota los resultados de los tres casos.

$k \in\{N, C, F\}$ donde $N$ denota la inversión individual, $C$ denota coinversión y $F$ denota regulación de costos totalmente distribuidos.

\footnotetext{
15 El uso del modelo de costos totalmente distribuidos se hace únicamente por simplicidad para poder comprender el problema. No obstante, se reconoce que posibles variaciones pueden ser importantes y se continuará avanzando en este aspecto en futuras investigaciones para modelar otras aproximaciones de costos tales como LRIC puro y LRIC+.

16 Para simplificar el modelo, los consumidores perciben que el servicio mejorado por calidad del entrante basado en servicios no difiere del establecido que es dueño de la infraestructura porque ellos operan sobre la misma infraestructura con el mismo grado de calidad. En otras palabras, los consumidores evalúan los servicios sin una preferencia específica por una firma.
} 


\section{ANÁLISIS ${ }^{17}$}

Los resultados de mercado cambian en relación a las diferentes aproximaciones de despliegue de infraestructura en los tres casos

Caso 1: Las dos firmas con infraestructura propia invierten en mejoras de calidad de manera separada (inversión individual)

En la etapa 2 del juego, la firma 1 y la firma 2 tienen sus problemas de maximización de beneficios, $\max _{q_{N 1}} \mathrm{y} \max \neq_{N 2}$ respectivamente. De (1.2) y (1.3), las condiciones de primer orden son

$$
\begin{aligned}
& \frac{\partial \pi_{N 1}}{\partial q_{N 1}}=a+\omega s_{N 1}-2 q_{N 1}-q_{N 2}=0 \\
& \frac{\partial \pi_{N 2}}{\partial 2}=a+\omega s_{N 2}-q_{N 1}-2 q_{N 2}=0
\end{aligned}
$$

Solucionando las condiciones de primer orden dadas arriba se encuentran los niveles de producción de equilibrio, tal y como se muestra a continuación.

$$
q_{N i}^{*}\left(s_{N i}, s_{N j}\right)=\frac{a}{3}+\frac{2}{3} \omega s_{N i}-\frac{1}{3} \omega s_{N j} \text { donde } i, j \in\{1,2\}, i \uparrow j
$$

Sustituyendo $q_{N i}^{*}$ en la función de beneficios (1.2) y (1.3) da como resultado la siguiente función de beneficios en la etapa 1

$$
\pi_{N i}\left(s_{N i}, s_{N j}\right)=\left(\frac{a}{3}+\frac{2}{3} \omega s_{N i}-\frac{1}{3} \omega s_{N j}\right)^{2}-\frac{\alpha_{i}}{2} s_{N i}^{2} \text { donde } i, j \in\{1,2\}, i \uparrow j
$$

El problema de la firma $i$ es $\max \neq_{N i}$. Diferenciando la función de beneficios en forma reducida con respecto a $\operatorname{los}^{s_{N i}}$ niveles asociados de mejoras en calidad da como resultado lo siguiente:

$$
\frac{\partial \pi_{N i}}{\partial s_{N i}}=\frac{4}{9} a \omega+\frac{8}{9} \omega^{2} s_{N i}-\frac{4}{9} \omega^{2} s_{N j}-\alpha_{i} s_{N i}=0
$$

donde $i, j \in\{1,2\}, i \uparrow j$.

En equilibrio, los niveles de mejoras de calidad de las dos firmas son

$$
\begin{aligned}
& s_{N 1}^{*}=\frac{12 a \omega \alpha_{2}-16 a w^{3}}{27 \alpha_{1} \alpha_{2}-24 \omega^{2} \alpha_{1}-24 \omega^{2} \alpha_{2}+16 \omega^{4}} \\
& s_{N 2}^{*}=\frac{12 a \omega \alpha_{1}-16 a w^{3}}{27 \alpha_{1} \alpha_{2}-24 \omega^{2} \alpha_{2}-24 \omega^{2} \alpha_{1}+16 \omega^{4}}
\end{aligned}
$$

\footnotetext{
${ }^{17}$ Las demostraciones de las proposiciones están disponibles a solicitud del interesado contactando directamente al autor.
} 


\section{Proposición 1}

En ausencia de acuerdos para desplegar infraestructura, la firma de costo bajo elige niveles de mejoras en calidad más altos y ofrece un mayor nivel de servicio con un precio más alto que la firma de costo alto, i.e. $s_{N 1}^{*}>s_{N 2}^{*}, q_{N 1}^{*}>q_{N 2}^{*}, p_{N 1}^{*}>p_{N 2}^{*}$. Finalmente, la firma de costo bajo obtiene mayores beneficios que la firma de costo alto, i.e. $\pi_{N 1}^{*}>\pi_{N 2}^{*}$.

La firma de costo bajo se convierte en dominante debido a los menores costos en los que incurre para mejorar la calidad. Debido a su eficiencia de costos, esta puede invertir más intensamente en mejorar la calidad atrayendo más consumidores y ganando una mayor participación de mercado al igual que mayores beneficios que la firma de costo alto cuyas mejoras en calidad son más costosas.

\section{Caso 2: Las dos firmas coinvierten}

Las firmas acuerdan la infraestructura total para el servicio avanzado. En la etapa 2, la firma 1 y la firma 2 tienen sus problemas de maximización, $\max _{q_{C 1}} \neq_{C 1}$ y $\max _{q_{C 2}} \neq_{C 2}$ respectivamente. De (1.4) y (1.5), las condiciones de primer orden son.

$$
\begin{aligned}
& \frac{\partial \pi_{C 1}}{\partial q_{C 1}}=a+\omega s_{C}-2 q_{C 1}-q_{C 2}=0 \\
& \frac{\partial \pi_{C 2}}{\partial q_{C 2}}=a+\omega s_{C}-q_{C 1}-2 q_{C 2}=0
\end{aligned}
$$

De (1.16) y (1.17), se pueden resolver las condiciones de primer orden y obtener los niveles de equilibrio del servicio como se muestra a continuación.

$$
q_{C i}^{*}\left(s_{C}\right)=\frac{a}{3}+\frac{1}{3} \omega s_{C} \text { donde } i, j \in\{1,2\}, i \uparrow j
$$

De vuelta a la etapa 1, sustituyendo (1.18) en las funciones de beneficios (1.4) y (1.5) da como resultado

$$
\begin{gathered}
\pi_{C 1}\left(s_{C}\right)=\left(\frac{a}{3}+\frac{1}{3} \omega s_{C}\right)^{2}-\beta \frac{\varnothing \alpha_{1}}{2} s_{C}^{2} \\
\pi_{C 2}\left(s_{C}\right)=\left(\frac{a}{3}+\frac{1}{3} \omega s_{C}\right)^{2}-(1-\beta) \frac{\varnothing \alpha_{1}}{2} s_{C}^{2}
\end{gathered}
$$

Las firmas entran en una negociación sobre los niveles mutuos de mejoras en calidad $\left(s_{C}\right)$ y división del costo total de inversión $(\beta)$. El resultado está basado sobre una solución de Nash. El resultado de la negociación soluciona el siguiente problema.

$$
\max _{\beta, s_{C}}\left(\pi_{C 1}-\pi_{N 1}^{*}\right)\left(\pi_{C 2}-\pi_{N 2}^{*}\right)
$$




$$
\begin{gathered}
\Psi \text { denota }\left(\pi_{C 1}-\pi_{N 1}^{*}\right)\left(\pi_{C 2}-\pi_{N 2}^{*}\right) . \\
\begin{array}{c}
\frac{\partial \cdot}{\partial \beta}=\left(\pi_{C 1}-\pi_{N 1}^{*}\right) \frac{\varnothing \alpha_{1}}{2} s_{C}^{2}+\left(\pi_{C 2}-\pi_{N 2}^{*}\right)\left(-\frac{\varnothing \alpha_{1}}{2} s_{C}^{2}\right)=0 \\
\pi_{C 1}-\pi_{N 1}^{*}=\pi_{C 2}-\pi_{N 2}^{*}(1.21) \\
\frac{\partial \cdot \cdot}{\partial s_{C}}=4 a \omega+4 \omega^{2} s_{C}-9 \varnothing \alpha_{1} s_{C}=0
\end{array}
\end{gathered}
$$

De (1.22), el nivel conjunto de mejora de calidad es

$$
s_{C}^{*}=\frac{4 a \omega}{9 \varnothing \alpha_{1}-4 \omega^{2}}
$$

\section{Proposición 2}

Por medio de la coinversión, la firma de costo bajo alcanza un acuerdo exitoso para pagar una fracción más pequeña del costo total de inversión de la infraestructura, como resultado genera mayores beneficios que la firma de costo alto, a pesar del mismo nivel de equilibrio de producción, i.e. $\beta^{*}<\frac{1}{2}, q_{C 1}^{*}=q_{C 2}^{*}, \pi_{C 1}^{*}>\pi_{C 2}^{*}$.

A la luz de la inversión individual, la firma de costo bajo esta en una posición dominante con niveles más altos tanto en beneficios como en mejoras de calidad. Una vez acordada la coinversión, las firmas obtienen la misma participación de mercado porque ambos servicios operan exactamente sobre la misma infraestructura compartida con el mismo nivel de mejoras en calidad. En la etapa 2, ambas firmas ofrecen niveles de producción simétricos en equilibrio. Sin embargo, durante la negociación de coinversión en la etapa 1, la firma de costo bajo tiene mayor poder para negociar una fracción más pequeńa del costo total de inversión. La firma de costo alto tiene que aceptar una proporción mucho más alta del costo total de inversión porque la negociación exitosa de coinversión finalmente trae mayores beneficios netos que la inversión individual. Como resultado, bajo la figura de coinversión, la firma de costo bajo obtiene mayores beneficios que la firma de costo alto, aunque sus servicios no son diferentes en términos de calidad mejorada y nivel del servicio desde la perspectiva de los consumidores en el mercado minorista.

Elaborando sobre este resultado, el mismo puede ser analizado de acuerdo con la regla de dividir la diferencia ${ }^{18}$. Cada firma acuerda extraer la misma cantidad de beneficio

\footnotetext{
${ }_{18}$ Muthoo (1999) ilustra un ejemplo de la regla de dividir la diferencia en el caso de dos jugadores negociando sobre una porción de pastel donde las funciones de utilidad de los jugadores son simplemente sus participaciones del pastel. La utilidad de un jugador puede ser reescrita como una función lineal de la utilidad del otro. Después de la negociación, ellos acuerdan obtener sus primeras porciones de manera equivalente a lo que ellos hubieran recibido si no se hubieran puesto de acuerdo, y adicionalmente acuerdan dividir el pastel de manera equivalente.
} 
como el que ganaría bajo inversión individual. Adicionalmente, ambas firmas llegan a un consenso para dividir de manera igualitaria el beneficio agregado adicional que ellas reciben al preferir coinvertir en vez haber invertido de manera individual. Aunque las firmas reciben participaciones iguales del beneficio agregado adicional, los beneficios de las dos firmas son diferentes. Esto es porque la firma de costo bajo tiene mayor poder de negociación para extraer una mayor cantidad de beneficio como consecuencia de su costo de oportunidad de coinvertir en vez de entrar a competir con inversión individual.

Los coinversionistas eligen un nivel mutuo de mejoras de calidad sin importar la repartición del costo de inversión. El nivel de mejoras de calidad directamente afecta el costo total de inversión. Sin embargo, ellos deciden sobre el nivel de mejoras de calidad únicamente en la base de la magnitud del costo total de inversión después de la coinversión y el precio de reserva de los consumidores. La asignación del costo total de inversión entre los coinversionistas depende de su poder de negociación. Ellos tienen menos incentivo de mejorar su calidad cuando el despliegue de infraestructura resulta en costos adicionales más sustanciales. Así, el nivel de equilibrio de la mejora de calidad se reduce cuando el parámetro de ajuste de costo de la compartición de infraestructura se incrementa.

Acceso a infraestructura mediante la aproximación de precio de acceso sin regular Cuando se permite que la firma con infraestructura propia de costo bajo fije el precio de acceso sin ninguna intervención por parte del regulador, esta rechazará la propuesta de despliegue de infraestructura o fijará un precio de acceso prohibitivo hasta que su rival basado en servicios no tenga participación de mercado y consecuentemente salga del mercado. Finalmente, la firma de costo bajo se convierte en un monopolista en la región e impone una limitación sobre el número de suscriptores lo que a su vez induce un aumento en el precio minorista. Por lo tanto, esta aproximación no es óptima en términos de bienestar del consumidor, aunque puede llevar a que el establecido realice más inversiones.

\section{Caso 3: Acceso a infraestructura bajo un régimen de costos totalmente distribuidos}

En la etapa 2, la firma 1 y la firma 2 elige su nivel de servicio para maximizar sus propios beneficios, $\max _{q_{F 1}} \neq_{F 1}$ y $\max _{q_{F 2}} \neq_{F 2}$ respectivamente. De (1.6) y (1.7), las condiciones de primer orden son

$$
\begin{aligned}
& \frac{\partial \pi_{F 1}}{\partial q_{F 1}}=a+\omega s_{F}-2 q_{F 1}-q_{F 2}-\left(\frac{\varnothing \alpha_{1}}{2} s_{F}^{2}\right)\left(\frac{q_{F 2}}{Q_{F}^{2}}\right)=0 \\
& \frac{\partial \pi_{F 2}}{\partial q_{F 2}}=a+\omega s_{F}-q_{F 1}-2 q_{F 2}-\left(\frac{\varnothing \alpha_{1}}{2} s_{F}^{2}\right)\left(\frac{q_{F 1}}{Q_{F}^{2}}\right)=0
\end{aligned}
$$

Para satisfacer (1.24) y (1.25), el equilibrio simétrico en esta etapa es 


$$
q_{F}^{*}\left(s_{F}\right)=q_{F 1}^{*}\left(s_{F}\right)=q_{F 2}^{*}\left(s_{F}\right)=\frac{a+\omega s_{F}}{6}+\sqrt{\frac{\left(a+\omega s_{F}\right)^{2}}{36}-\frac{\varnothing \alpha_{1} s_{F}^{2}}{24}}
$$

Bajo el régimen regulatorio de costos totalmente distribuidos, la firma 1 elige el grado de mejoras en calidad en la etapa 1 para resolver su problema, $\max _{s_{F}} \neq_{F 1}$. Después de sustituir (1.26) en (1.6), se puede diferenciar la función de beneficios en forma reducida de la firma 1 y obtener la siguiente ecuación.

$$
\frac{\partial \pi_{F 1}}{\partial s_{F}}=\left(a+\omega s_{F}-4 q_{F 1}^{*}\right) \frac{\partial q_{F 1}^{*}}{\partial s_{F}}+\omega q_{F 1}^{*}-\frac{\varnothing \alpha_{1} s_{F}}{2}=0
$$

El nivel de equilibrio de la mejora de calidad $\left(s_{F}^{*}\right)$ no puede ser expresado de manera explícita en forma cerrada. Sin embargo, se puede comparar con los resultados de los otros casos, como se verá en la siguiente sección.

\section{Proposición 3}

Bajo el régimen regulatorio de costos totalmente distribuidos, la participación de mercado y el beneficio de la firma basada en infraestructura de costo bajo son iguales a los de la firma basada en servicios de costo alto que solicita acceso de red a la firma de costo bajo, i.e. $q_{F 1}^{*}=q_{F 2}^{*} y \pi_{F 1}^{*}=\pi_{F 2}^{*}$.

Bajo la regulación de costos totalmente distribuidos, la firma de costo bajo es regulada para permitir que su rival basado en servicios acceda a su infraestructura. En el mercado minorista, las dos firmas compiten la una con la otra ofreciendo exactamente el mismo nivel de servicio con calidad mejorada que funciona sobre la misma infraestructura ${ }^{19}$. Así, las dos firmas son iguales en participación de mercado y beneficios.

\section{Regímenes regulatorios óptimos}

La tabla 1 muestra las comparaciones de los resultados de equilibrio de mercado bajo diferentes regímenes regulatorios con el fin de encontrar la regulación/desregulación más efectiva para el despliegue de tecnología avanzada en diferentes facetas de mejoras de calidad, la oferta de servicios y el bienestar del consumidor en general.

\footnotetext{
19 En este modelo, se asume que no existe diferenciación horizontal del producto entre proveedores de servicio. Los consumidores únicamente consideran precio y calidad del servicio sin fijarse en la marca.
} 
Tabla 1. Resultados de equilibrio de mercado en comparación

\begin{tabular}{lc}
\hline \multicolumn{1}{c}{ Escenario } & Grado de mejora de calidad \\
\hline Caso 1: Inversión individual & $s_{N 1}^{*}=\frac{12 a \omega \alpha_{2}-16 a \omega^{3}}{27 \alpha_{1} \alpha_{2}-24 \omega^{2} \alpha_{1}-24 \omega^{2} \alpha_{2}+16 \omega^{4}}$ \\
& $s_{N 2}^{*}=\frac{12 a \omega \alpha_{1}-16 a \omega^{3}}{27 \alpha_{1} \alpha_{2}-24 \omega^{2} \alpha_{2}-24 \omega^{2} \alpha_{1}+16 \omega^{4}}$ \\
$\begin{array}{l}\text { Caso 2: Despliegue de infraestructura por } \\
\text { coinversión }\end{array}$ & $s_{C}^{*}=\frac{4 a \omega}{9 \varnothing \alpha_{1}-4 \omega^{2}}$ \\
$\begin{array}{l}\text { Caso 3: Acceso a infraestructura bajo el régimen } \\
\text { regulatorio de costos totalmente distribuidos }\end{array}$ & $\mathrm{n} / \mathrm{a}$ \\
\hline
\end{tabular}

\begin{tabular}{cc}
\hline Escenario & Nivel de producción de la industria \\
\hline
\end{tabular}

Caso 1: Inversión individual

$$
Q_{N}^{*}=\frac{2 a}{3}+\frac{\omega s_{N 1}^{*}}{3}+\frac{\omega s_{N 2}^{*}}{3}
$$

Caso 2: Despliegue de infraestructura por coinversión

$$
Q_{C}^{*}=\frac{2\left(a+\omega s_{C}^{*}\right)}{3}
$$

Caso 3: Acceso a infraestructura bajo el régimen regulatorio de costos totalmente distribuidos

$$
Q_{F}^{*}=\frac{\left(a+\omega s_{F}^{*}\right)}{3}+2 \sqrt{\frac{\left(a+\omega s_{F}^{*}\right)^{2}}{36}-\frac{\varnothing \alpha_{1} s_{F}^{* 2}}{24}}
$$

\section{Escenario}

Caso 1: Inversión individual

Caso 2: Despliegue de infraestructura por coinversión

Caso 3: Acceso a infraestructura bajo el régimen regulatorio de costos totalmente distribuidos

$$
C S_{F}=\left(\frac{1}{2}\right)\left(\frac{\left(a+\omega s_{F}^{*}\right)}{3}+2 \sqrt{\frac{\left(a+\omega s_{F}^{*}\right)^{2}}{36}-\frac{\varnothing \alpha_{1} s_{F}^{*_{2}}}{24}}\right)^{2}
$$

Nota: n/a es el resultado de equilibrio que no puede ser expresado en forma cerrado.

Los resultados de equilibrio varían de acuerdo con las circunstancias. Este estudio está dirigido a analizar la figura de coinversión, así que el resultado de coinversión será comparado con otros casos como referente.

\section{- Coinversión vs. inversión individual}

Esta sección resalta las comparaciones entre los resultados de coinversión e inversión individual 


\section{Proposición 4}

En comparación a la figura de inversión individual, los coinversionistas acuerdan cambiar el nivel de mejoras en calidad de acuerdo con su estructura ajustada de costos producto de la compartición de infraestructura.

(i) Si el parámetro de ajuste de costos es muy bajo, $0<\varnothing<\frac{9 \alpha_{1} \Delta_{\alpha}-8 \omega^{2} \Delta_{\alpha}}{9 \alpha_{1} \Delta_{\alpha}-12 \omega^{2}}$, los coinversionistas acuerdan incrementar el nivel mutuo de mejoras en calidad, i.e. $s_{N 2}^{*}<s_{N 1}^{*}<s_{C}^{*}$.

(ii) Si el parámetro de ajuste de costos es moderado, $\frac{9 \alpha_{1} \Delta_{\alpha}-8 \omega^{2}-4 \omega^{2} \Delta_{\alpha}}{9 \alpha_{1} \Delta_{\alpha}-12 \omega^{2}} \leq \varnothing \leq$ $\frac{9 \alpha_{1} \Delta_{\alpha}-8 \omega^{2} \Delta_{\alpha}-4 \omega^{2}}{9 \alpha_{1}-12 \omega^{2}}$, el nivel mutuo de mejoras en calidad se ubica entre el de la firma de alto costo y el de la firma de costo alto con inversión individual, i.e. $s_{N 2}^{*}<s_{C}^{*}<s_{N 1}^{*}$.

(iii) Finalmente, si el parámetro de ajuste de costos es suficientemente alto, $\varnothing>\frac{9 \alpha_{1} \Delta_{\alpha}-8 \omega^{2} \Delta_{\alpha}-4 \omega^{2}}{9 \alpha_{1}-12 \omega^{2}}$, los coinversionistas reducen el nivel mutuo de mejoras en calidad, i.e. $s_{C}^{*}<s_{N 2}^{*}<s_{N 1}^{*}$.

Cuando los beneficios de la reducción de costos, producto de la compartición de infraestructura son lo suficientemente significativos, los coinversionistas ofrecen un nivel más alto de mejoras en calidad en comparación con el resultado de equilibrio de la inversión individual. El ahorro en costos de la compartición de infraestructura puede estimular mejoras mutuas en la calidad del servicio. Sin embargo, si la compartición de infraestructura requiere equipo adicional y operación especial que genera costos incrementales sustanciales, los coinversionistas acordaran elegir un nivel más bajo de mejoras mutuas en calidad comparado con el resultado de inversión individual. Esto es porque los coinversionistas incurren en costos sustanciales de inversión después del acuerdo. Por lo tanto, ellos se ponen de acuerdo en reducir la competencia disminuyendo el grado mutuo de mejoras en calidad con el fin de recibir más beneficios que bajo el caso de inversión individual.

La influencia del parámetro de ajuste de costos de despliegue de infraestructura $(\varnothing)$ en la negociación entre coinversionistas

Para subrayar el efecto del parámetro de ajuste de costos de compartición de infraestructura $(\varnothing)$ sobre el incentivo de las firmas para mejorar su calidad, se asume que las dos firmas son simétricas es su estructura de costos de inversión $\left(\alpha_{1}=\alpha_{2}\right)$. En esta situación, el parámetro de ajuste de costos $(\varnothing)$ tiene un efecto significativo sobre la negociación sobre el nivel de mejoras en calidad bajo coinversión tal y como se presenta a continuación. 


\section{Corolario 1}

Cuando las dos firmas son simétricas en la estructura de costos de inversión, el resultado de equilibrio simétrico ocurre, i.e. $s_{N 1}^{*}=s_{N 2}^{*}$. Cuando $0<\varnothing<1, \varnothing=1, o \varnothing>1$, el nivel mutuo de mejoras en calidad bajo el acuerdo de coinversión es más alto que, igual a, o menor que aquellos bajo inversión individual respectivamente. Cada coinversionista acuerda pagar la mitad del costo total de inversión de la compartición de infraestructura, i.e. $\beta=\frac{1}{2}$.

En el caso especial de simetría en la estructura de costos, el parámetro de ajuste de $\operatorname{costos}(\varnothing)$ desempeña un papel crucial en las decisiones de la firma sobre las mejoras en calidad. Cuando no se comparte infraestructura, ambas firmas eligen el mismo nivel de mejoras en calidad y reciben el mismo beneficio. Las dos tienen el mismo poder de negociación cuando entran a negociar la coinversión. Como resultado, el costo total de inversión se divide de manera igual entre los coinversionistas. Cuando el acuerdo para desplegar infraestructura no induce a un aumento en costos $(\varnothing)$, los coinversionistas pagan exactamente la misma suma en costos de inversión y el problema de decisión de los coinversionistas es idéntico al problema de decisión de los inversionistas individuales. Por lo tanto, después de acordada la coinversión, las firmas no cambian sus niveles de equilibrio de mejoras en calidad. Sin embargo, cuando la compartición de infraestructura involucra costos adicionales $(\varnothing>0)$, los coinversionistas acuerdan reducir el grado de mejoras en calidad en respuesta al costo adicional. En contraste, cuando el despliegue de infraestructura resulta en ahorro de $\operatorname{costos}(0<\varnothing<1)$, el costo de mejoras en calidad es menor una vez realizada la coinversión. Los coinversionistas encuentran más rentable aumentar la calidad del servicio con el propósito de atraer más consumidores. Así, en este contexto de estructura de costos simétrica, aunque las firmas pueden alivianar su propia carga de costo de inversión a través de la coinversión, no se garantiza que las firmas tengan incentivo alguno de aumentar sus niveles mutuos de mejoras en calidad. Cuando la coinversión genera costos adicionales para facilitar el despliegue de infraestructura, los coinversionistas tácitamente coluden para disminuir los niveles mutuos de mejoras en calidad a través de la negociación de la coinversión.

De manera similar, de regreso al modelo principal de estructura de costos asimétricos como se vio en la proposición 4, la colusión tacita para reducir el nivel mutuo de mejoras en calidad es probable que ocurra cuando la coinversión genera una cantidad considerable de costos de inversión para implementar el despliegue de infraestructura.

Adicionalmente, el parámetro de ajuste de costos del despliegue de la infraestructura $(\varnothing)$ también desempeńa un papel crucial en el equilibrio de la producción de la industria y el bienestar del consumidor bajo coinversión, tal y como se establece en la siguiente proposición. 


\section{Proposición 5}

El nivel de equilibrio de producción de la industria y el cambio en el excedente del consumidor de acuerdo con la estructura de costos de los coinversionistas.

Si $0<\varnothing \leq \frac{6 \alpha_{1} \Delta_{\alpha}-4 \omega^{2} \Delta_{\alpha}-4 \omega^{2}}{3 \alpha_{1}+3 \alpha_{1} \Delta_{\alpha}-8 \omega^{2}}$, el nivel de equilibrio de producción de la industria y el excedente del consumidor son más altos que o iguales a los que se obtienen bajo inversión individual, i.e. $Q_{C}^{*} \geq Q_{N}^{*}$ y $C S_{C} \geq C S_{N}$. De manera opuesta, si $\varnothing>\frac{6 \alpha_{1} \Delta_{\alpha}-4 \omega^{2} \Delta_{\alpha}-4 \omega^{2}}{3 \alpha_{1}+3 \alpha_{1} \Delta_{\alpha}-8 \omega^{2}}$, el nivel de equilibrio de producción de la industria y el excedente del consumidor son menores a los de la inversión individual, i.e. $Q_{C}^{*}<Q_{N}^{*}$ y $C S_{C}<C S_{N}$.

Cuando los coinversionistas encuentran que la coinversión lleva a considerables costos incrementales de despliegue de infraestructura $\left(\varnothing>\frac{6 \alpha_{1} \Delta_{\alpha}-4 \omega^{2} \Delta_{\alpha}-4 \omega^{2}}{3 \alpha_{1}+3 \alpha_{1} \Delta_{\alpha}-8 \omega^{2}}\right)$, estos deciden reducir el número total de suscriptores en el área. De acuerdo con la proposición 4, y en comparación a la inversión individual, si el parámetro de ajuste de costos es muy alto, los coinversionistas se enfocan en ofrecer un nivel bajo de mejoras en calidad para ahorrar en costos. Aunque esta estrategia obliga a los coinversionistas a renunciar a algunos suscriptores, la misma puede aumentar los beneficios de los coinversionistas desalentando la competencia en torno a la calidad. Consecuentemente, cuando los coinversionistas incurren en costos altos de inversión después del despliegue de infraestructura, ellos coluden para reducir la competencia ofreciendo servicios con una menor calidad. Esto causa una reducción en el excedente del consumidor. En contraste, si la coinversión lleva a ahorros en costos o solo a un aumento reducido de los costos, $0<\varnothing<\frac{6 \alpha_{1} \Delta_{\alpha}-4 \omega^{2} \Delta_{\alpha}-4 \omega^{2}}{3 \alpha_{1}+3 \alpha_{1} \Delta_{\alpha}-8 \omega^{2}}$, los coinversionistas tienen un incentivo a aumentar el número de suscriptores en el mercado. Comparado a la situación de inversión individual, todos los consumidores activos en el caso de un parámetro de ajuste de costos bajo y muchos de los consumidores activos en el caso de un parámetro de ajuste de costos moderado se benefician de niveles más altos de mejoras en calidad. Así, la base de consumidores se expande y el excedente del consumidor aumenta consecuentemente.

\section{Coinversión $v s$. regulación de costos totalmente distribuidos}

La comparación de las dos aproximaciones de despliegue de infraestructura es hecha en términos de mejoras de calidad, la oferta de servicios, precio y excedente del consumidor. 


\section{Proposición 6}

El despliegue de infraestructura bajo una regulación de costos totalmente distribuidos genera un mayor nivel de mejoras en calidad, pero un nivel menor de producción de la industria con precios minoristas más altos que los que se obtienen bajo el resultado de coinversión, i.e. $s_{F}^{*}>s_{C}^{*}, Q_{F}^{*}<Q_{C}^{*} y p_{F}^{*}>p_{C}^{*}$. En general, la regulación genera un menor excedente del consumidor que la coinversión, i.e. $C S_{F}<C S_{C}$.

En ausencia de diferenciación horizontal en preferencia de marca, la competencia entre la firma basada en infraestructura y su rival basado en servicios en el mercado minorista termina con participaciones de mercado iguales. Entonces, ellas comparten la mitad del costo total de inversión bajo la regulación de costos totalmente distribuidos. Sin embargo, la firma de costo bajo basada en infraestructura invierte en toda la infraestructura y tiene el derecho de unilateralmente decidir sobre el nivel de mejoras en calidad. Comparado con la coinversión, la firma basada en infraestructura que es más rentable atrae consumidores aumentando el nivel de mejoras en calidad lo cual le permite cobrar un precio minorista más alto bajo esta regulación. A pesar de un servicio avanzado más atractivo, el excedente del consumidor bajo regulación de costos totalmente distribuidos es menor que bajo coinversión. Esto es porque la firma basada en infraestructura tiene por objetivo limitar el producto de la industria para poder maximizar su propio beneficio.

\section{Los efectos del despliegue de infraestructura sobre la rentabilidad de las firmas}

Esta sección discute los efectos de las diferentes aproximaciones de despliegue de infraestructura sobre la rentabilidad de las firmas

\section{Proposición 7 a}

Ambas firmas basadas en infraestructura alcanzan un acuerdo exitoso de coinversión porque sus beneficios de coinvertir son más altos que los beneficios obtenidos bajo la figura de inversión individual de acuerdo con el beneficio del parámetro de ajuste de costos de despliegue de infraestructura $(\varnothing)$, i.e. $\pi_{C 1}^{*}>\pi_{N 1}^{*} y \pi_{C 2}^{*}>\pi_{N 2}^{*}$.

Bajo el supuesto de que el costo total de la inversión individual es mayor que el de despliegue conjunto de infraestructura, las firmas con infraestructura propia de bajo costo encuentran más rentable ponerse de acuerdo en coinvertir que en construir su propia infraestructura de manera separada. De manera similar, la firma de alto costo también se beneficia de la infraestructura de bajo costo proporcionada por la firma de bajo costo. Por lo tanto, las dos firmas llegan a un acuerdo exitoso sobre la coinversión en vez de realizar sus inversiones separadamente. 


\section{Proposición $7 \mathrm{~b}$}

(i) La comparación entre los beneficios de la firma de costo bajo con coinversión y bajo regulación de costos totalmente distribuidos es ambigua. Si el parámetro de asimetría de costos es muy bajo ( $\Delta_{\alpha}$ se aproxima a uno), el beneficio de la firma de bajo costo bajo la regulación de costos totalmente distribuidos es más alto que bajo coinversión, i.e. $\pi_{F 1}^{*}>$ $\pi_{C 1}^{*}$. De manera opuesta, si el parámetro de asimetría de costos $\left(\Delta_{\alpha}\right)$ es suficientemente alto, los beneficios de la firma de bajo costo bajo la regulación de costos totalmente distribuidos tienen una tendencia a ser menores que bajo coinversión, i.e. $\pi_{F 1}^{*}<\pi_{C 1}^{*}$.

(ii) La firma con infraestructura propia de costo bajo prefiere la figura de despliegue de infraestructura mediante la aproximación de precio de acceso sin regular que la de coinversión, i.e. $\pi_{A 1}^{*}>\pi_{C 1}^{*}$.

Cuando el regulador interviene a través de la figura de regulación de costos totalmente distribuidos, la firma con infraestructura propia de costo bajo puede ganar o perder beneficios debido a esta regulación. Cuando la asimetría de costos no es tan significativa, el poder de negociación de la firma de costo bajo es menor. Su beneficio de equilibrio bajo coinversión es menor que bajo regulación de costos totalmente distribuidos. Esto es porque bajo la regulación de costos totalmente distribuidos, la firma de costo bajo tiene el derecho de unilateralmente elegir el nivel de mejoras en calidad para maximizar su propio beneficio. Mientras tanto, bajo coinversión, la firma de costo bajo tiene que negociar con el otro coinversionista para determinar el nivel mutuo de mejoras en calidad teniendo en cuenta los intereses de su rival. De otro lado, cuando la asimetría de costos es lo suficientemente significativa, los beneficios de la firma de costo bajo con coinversión son mayores que bajo la regulación de costos totalmente distribuidos. La firma de costo bajo tiene un poder claro en la negociación de la coinversión. Esta paga una proporción considerablemente menor del costo total de inversión bajo coinversión, mientras que tiene que compartir la mitad del costo total de inversión bajo la regulación de costos totalmente distribuidos de acuerdo con la igualdad de niveles de producción en equilibrio. Como resultado, su beneficio bajo coinversión probablemente es mayor que bajo la regulación de costos totalmente distribuidos.

Cuando se permite que la firma basada en infraestructura de costo bajo fije el precio de acceso, esta puede encerrar el mercado cobrando un precio de acceso excesivamente alto. El beneficio monopolístico de la firma de costo bajo en este régimen regulatorio es definitivamente más alto que bajo coinversión en un duopolio.

\section{Proposición 7c}

Bajo una figura de entrada basada en servicios, la firma de alto costo prefiere este tipo de entrada bajo la regulación de costos totalmente distribuidos que coinversión, inversión individual y entrada basada en servicios por el precio de acceso sin regular respectivamente porque su beneficio bajo la regulación de costos totalmente distribuidos es el más alto, i.e. $\pi_{F 2}^{*}>\pi_{N 2}^{*}>\pi_{A 2}^{*}$. 
Cuando el regulador interviene con la regulación de costos totalmente distribuidos, la firma de alto costo elige una entrada basada en servicios en vez de coinversión para poder aumentar su beneficio. Sin embargo, cuando se permite que la firma basada en infraestructura de costo bajo fije el precio de acceso, la firma de costo alto será excluida del mercado si esta pide acceso de red a la firma de costo bajo. Por lo tanto, la firma de costo alto prefiere la regulación de costos totalmente distribuidos.

\section{IMPLICACIONES DE POLÍTICA}

Entre los escenarios discutidos para el despliegue de infraestructura, la aproximación óptima depende de cuál sea la prioridad para el regulador. La coinversión provoca que haya menores incentivos para mejorar la calidad, pero puede expandir el tamaño de la base de suscriptores y resultar en un excedente del consumidor mucho más alto del que se obtiene bajo la regulación de costos totalmente distribuidos. La comparación entre mejoras de calidad y excedente del consumidor bajo coinversión y bajo inversión individual es ambigua de acuerdo con el grado de beneficio del ahorro de costos a través del acuerdo de despliegue de infraestructura. Si el beneficio de ahorro de costos es sustancial, la calidad del servicio mejorará significativamente con la estructura de costos eficiente y el bienestar del consumidor también mejorará. Por lo tanto, se sugiere que el regulador apoye las negociaciones de coinversión, únicamente si el acuerdo de despliegue de infraestructura da como resultado un beneficio sustancial en reducción de costos. De otro modo, es probable que se presenten escenarios de colusión en mejoras de calidad y que la inversión individual sea la figura más apropiada en términos de bienestar del consumidor. De hecho, los acuerdos de coinversión pueden ser vistos como acuerdos entre competidores y pueden constituir "fusiones" y/o "concentraciones". Estos podrían por lo tanto ser potencialmente de interés para las autoridades de competencia quienes estarían inclinadas a entender las implicaciones de tales acuerdos para la competencia.

\section{CONCLUSIONES}

Este estudio examina los resultados de equilibrio bajo la presencia de compartición de infraestructura mediante diferentes aproximaciones. Las dos firmas asimétricas tienen diferentes estructuras de costos de inversión en calidad. Ellas eligen el grado de mejoras en calidad que pueden aumentar la utilidad del consumidor para entonces determinar la base de consumidores a ser atendida. Bajo la figura de inversión individual, la firma de costo bajo ofrece niveles altos de mejoras de calidad, producción y correspondiente precio minorista que la firma de costo alto. La firma de costo bajo está en la mejor posición con beneficios más altos que la firma de costo alto debido a su ventaja de menores costos.

Las dos firmas acuerdan coinvertir en desplegar infraestructura en vez de invertir individualmente porque ellas pueden aumentar sus propios beneficios bajo cooperación. 
En concordancia con la solución de negociación de Nash, la firma de costo bajo con mayor poder de negociación enfrentará menores costos de inversión que la firma de costo alto. Como resultado, la firma de costo bajo recibe un beneficio más alto. Sin embargo, la comparación entre los resultados de coinversión e inversión individual es ambiguo. Cuando la compartición de infraestructura resulta en un beneficio sustancial de reducción de costos (respectivamente la compartición de infraestructura lleva a una cantidad considerable de costos incrementales), el nivel de equilibrio de la mejora de calidad, producción de la firma, producción de la industria y excedente del consumidor bajo coinversión son mayores que (respectivamente menores que) bajo inversión individual. Por lo tanto, la coinversión puede reducir la competencia si la compartición de infraestructura no genera ahorros significativos. En esta situación, la negociación sobre coinversión se convierte en colusión más que promover las mejoras en calidad y excedente del consumidor.

Después de la intervención regulatoria bajo el esquema de costos totalmente distribuidos, la firma basada en servicios de costo alto tiene un incentivo a no coinvertir en infraestructura compartida, sino buscar acceso de red de la firma con infraestructura propia de costo bajo. Así, las firmas tienen igual participación de mercado y beneficios porque ambas operan sobre la misma infraestructura de red y ofrecen servicios idénticos con la misma calidad. Comparado a la situación de coinversión, la firma de costo alto puede aumentar sus beneficios bajo esta regulación porque enfrenta menores costos de inversión. Cuando las estructuras de costos de ambas firmas son levemente diferentes (significativamente diferentes), los beneficios de la firma de costo bajo aumentan (tienen una tendencia a disminuir). La firma de costo bajo decide ofrecer un nivel de mejoras en calidad más alto, pero atiende un grupo menor de suscriptores de lo que haría bajo coinversión. Como resultado, el precio minorista bajo esta regulación es más alto que bajo coinversión. En general, la regulación de costos totalmente distribuidos reduce el excedente del consumidor.

El regulador de telecomunicaciones debería monitorear los acuerdos de coinversión, especialmente cuando la compartición de infraestructura no da como resultado un ahorro de costos sustancial. En esta situación, las firmas pueden coinvertir para desalentar la competencia haciendo que el excedente del consumidor sea menor que bajo la situación de inversión individual. En adición, la regulación de costos totalmente distribuidos es más efectiva para promover la entrada basada en servicios que la coinversión y la aproximación de precios de acceso sin regular. Esta regulación también puede soportar la firma basada en infraestructura de bajo costo si las dos firmas son levemente diferentes en estructura de costos. De manera opuesta, si la estructura de costos de las dos firmas es significativamente diferente, es probable que esta regulación ponga en riesgo a la firma de bajo costo. Esto es porque su poder de negociación sobre la asignación de costos es mucho menor bajo el régimen de costos totalmente distribuidos que bajo coinversión. La regulación de costos totalmente distribuidos puede estimular las mejoras de calidad 
de la firma de costo bajo y acomodar al entrante basado en servicios de costo alto, pero puede provocar una reducción en la base de consumidores y en el excedente del consumidor. El regulador debería tomar esto en consideración si planea imponer esta regulación para poder facilitar la entrada basada en servicios de la firma de costo alto en vez de promover la negociación de coinversión. La regulación optima depende de lo que el regulador considere prioritario.

Este estudio analiza la competencia entre dos firmas que cuentan con información completa sobre sus estructuras de costos. Sin embargo, las firmas pueden tener información incompleta sobre los costos de sus rivales. Adicionalmente, este estudio está basado sobre el supuesto de demanda unitaria. Las firmas deciden sobre la mejora de calidad de solo un servicio en la línea de producción. Investigación adicional puede ser realizada para profundizar sobre el tema de la información incompleta sobre los tipos de costos de las firmas. También estudios futuros pueden ser extendidos al caso de múltiples firmas, demanda variable y las decisiones de las firmas sobre la calidad de un rango mucho más amplio de productos para atender diferentes grupos de consumidores. Adicionalmente, otras aproximaciones de despliegue de infraestructura pueden ser de interés tales como las asociaciones público-privadas, entendiendo estas como figuras de coinversión entre el sector público y el sector privado. Por último, se podría cambiar el supuesto de costos totalmente distribuidos por otras aproximaciones de costos tales como costos incrementales de largo plazo.

\section{ANEXO}

\section{Definiciones de términos clave}

Coinversión: inversión conjunta que realizan dos o más operadores para desplegar infraestructura, siendo los operadores dueńos de dicha infraestructura.

Costos totalmente distribuidos: un método contable para distribuir todos los costos entre los diferentes productos y servicios de una firma, por lo tanto, este método puede incluir costos que no están directamente asociados con un producto o servicio en particular.

Producción: para los términos de este estudio se entiende como capacidad instalada

Entrada basada en infraestructura: se refiere al hecho cuando un operador construye su propia infraestructura para entrar al mercado.

Entrada basada en servicios: se da cuando un operador arrienda la infraestructura del establecido para poder competirle a este en el servicio final que se ofrece al consumidor. Redes de nueva generación: redes de acceso a internet, principalmente fibra óptica, que alcanzan velocidades de más de $100 \mathrm{Mbps}$ 


\section{REFERENCIAS BIBLIOGRÁFICAS}

Bacache, Maya, Bourreau, Marc y Gaudin, Germain (2014). Dynamic entry and investment in new infrastructures: Empirical evidence from the fixed broadband industry. Review of Industrial Organization, 44(2), 179-209.

Beckman, Claes y Smith, Arthur (2005). Shared networks: making wireless communication affordable. Wireless Communications, IEEE, 12(2), 78-85.

Bouckaert, Jan, van Dijk, Theon. y Verboven, Frank (2010). Access regulation, competition, and broadband penetration: An international study. Telecommunications Policy, 34(11), 661-671.

Bourreau, Marc, Cambini, Carlo y Doğan, Pinar (2012). Access pricing, competition, and incentives to migrate from "old" to "new" technology. International Journal of Industrial Organization, 30(6), 713-723.

Bourreau, Marc, Cambini, Carlo y Hoernig, Steffen (2012). Ex ante regulation and co-investment in the transition to next generation access. Telecommunications Policy, 36(5), 399-406.

Bourreau, Marc, Cambini, Carlo y Hoernig, Steffen (2015). Geographic access markets and investments. Information Economics and Policy, 31, 13-21.

Briglauer, Wolfgang, Ecker, Georg y Gugler, Klaus (2013). The impact of infrastructure and service-based competition on the deployment of next generation access networks: Recent evidence from the European member states. Information Economics and Policy, 25(3), 142-153.

Briglauer, Wolfgang, Gugler, Klaus. and Haxhimusa, Adhurim (2015). Facility- and servicebased competition and investment in fixed broadband networks: Lessons from a decade of access regulations in the European Union member states. Telecommunications Policy, 40(8), 729-742.

Cambini, Carlo y Jiang, Yanyan (2009). Broadband investment and regulation: A literature review. Telecommunications Policy, 33(10), 559-574.

Cambini, Carlo y Silvestri, Virginia (2013). Investment sharing in broadband networks. Telecommunications Policy, 37(10), 861-878.

Cambini, Carlo y Valletti, Tommaso (2003). Network competition with price discrimination: 'Bill-and-Keep' is not so bad after all. Economics Letters, 81, 205-213.

Cave, Martin (2006). Encouraging infrastructure competition via the ladder of investment. Telecommunications Policy, 30(3), 223-237.

Cave, Martin y Vogelsang, Ingo (2003). How access pricing and entry interact. Telecommunications Policy, 27(10), 717-727.

Crandall, Robert, Eisenach, Jeffrey y Ingraham, Allan (2013). The long-run effects of copperloop unbundling y the implications for fiber. Telecommunications Policy, 37(4), 262-281.

Foros, Øystein (2004). Strategic investments with spillovers, vertical integration and foreclosure in the broadband access market. International Journal of Industrial Organization, 22(1), $1-24$.

Foros, Øystein, Hansen, Bjorn y Sand, Jan (2002). Demand-side spillovers and semi-collusion in the mobile communications market. Journal of Industry, Competition and Trade, 2(3), 259-278.

Grajek, Michal y Röller, Lars Hendrick (2012). Regulation and investment in network industries: Evidence from European telecoms. Journal of Law and Economics, 55(1), 189-216.

Kotakorpi, Kaisa (2006). Access price regulation, investment and entry in telecommunications. International Journal of Industrial Organization, 24(5), 1013-1020. 
Krämer, Jan y Vogelsang, Ingo (2016). Co-Investments and Tacit Collusion in Regulated Network Industries: Experimental Evidence. Review of Network Economics, 15(1), 35-61. Mussa, Michael y Rosen, Sherwin (1978). Monopoly and product quality. Journal of Economic Theory, 18(2), 301-317.

Muthoo, Abhinay (1999). Bargaining theory with applications. Cambridge: Cambridge University Press.

Nitsche, Rainer y Wiethaus, Lars (2011). Access regulation and investment in next generation networks - A ranking of regulatory regimes. International Journal of Industrial Organization, 29(2), 263-272.

Song, Young-Keun, Zo, Hang-Jung y Lee, Sung-Joo (2012). Analyzing the economic effect of mobile network sharing in Korea. ETRI Journal, 34(3), 308-318.

Valletti, Tommaso (2000). Minimum quality standards under Cournot competition. Journal of Regulatory Economics, 18(3), 235-245.

Vareda, Joao (2010). Access regulation and the incumbent investment in quality-upgrades and in cost-reduction. Telecommunications Policy, 34(11), 697-710.

Documento recibido el 16 de agosto de 2017 y aprobado el 24 de noviembre de 2017 